\title{
Time-Effect of Donor and Recipient Characteristics on Graft Survival after Kidney Transplantation
}

\author{
jingyan Yang, DrPH, MHS ${ }^{1, *}$ Christine L. Sardo Molmenti, MPH, $\mathrm{PhD}^{2, *} \quad$ Joaquin Cagliani, MD, $\mathrm{PhD}^{3}$ (1) \\ Harish Datta, BS ${ }^{2}$ Elliot Grodstein, MD $^{3}$ Rehana Rasul, MPH ${ }^{2}$ Horacio Rilo, MD \\ Lewis W. Teperman, MD ${ }^{3}$ Ernesto P. Molmenti, MD, PhD, MBA ${ }^{3}$
}

${ }^{1}$ Department of Epidemiology, Mailman School of Public Health,

Address for correspondence Ernesto P. Molmenti, MD, PhD, MBA, Columbia University, New York

2 Department of Occupational Medicine, Epidemiology and Department of Surgery, Northwell Health, 400 Community Drive, Prevention, Northwell Health, Great Neck, New York

${ }^{3}$ Department of Surgery, Northwell Health, Manhasset, New York Manhasset, New York 11030 (e-mail: emolment@northwell.edu).

Int J Angiol 2019;28:249-254.

\begin{abstract}
Keywords

- renal transplantation

- kidney transplantation

- KAS

- UNOS

- graft survival

- organ procurement

- recipient characteristics

The kidney allocation system (KAS) is based on quality-based "longevity matching" strategies that provide only a momentary snapshot of expected outcomes at the time of transplantation. The purpose of our study was to define on a continuous timeline the relative and mutual interactions of donor and recipient characteristics on graft survival. Total 39,108 subjects who underwent kidney transplant between October 25, 1999 and January 1, 2007 were identified in the United Network for Organ Sharing dataset. Our primary outcome was graft survival. Time-dependent receiver operating characteristic (ROC) curves and area under time-dependent ROC curve (AUC) were used to compare the predictive ability of the two allocation systems.

During the first year after transplantation, both donor and recipient models showed identical relevance. From the first to the sixth years, although the two ROC curves were nearly identical, the donor model outweighed the recipient model. Both models intersected again at the sixth year. From that time onward, the ROC curve for recipient characteristics model predominated over the ROC curve for donor characteristics model. The predictive value of the recipient model (AUC $=0.752$ ) was greater than that of the donor model (AUC $=0.673$ )

We hope that this model will provide additional guidance and risk stratification to further optimize organ allocation based on the dynamic interaction of both donor and recipient characteristics over time.
\end{abstract}

Multiple models have been developed to evaluate the effect of various donor and recipient characteristics on outcome after kidney transplantation. The current kidney allocation system (KAS) is based on the Kidney Donor Profile Index (KDPI) and the Estimated Post-Transplant Survival (EPTS). The KDPI determines the quality of each individual deceased donor kidney relative to the other organs recovered from such donors. ${ }^{1}$ The EPTS score is used to identify

Both the authors have equal contribution. candidates with the best potential survival. ${ }^{2}$ Currently, recipients with EPTS $\leq 20 \%$ are matched with organs from donors with KDPI $\leq 20 \%$.

Unfortunately, these quality-based "longevity matching" strategies provide only a momentary snapshot of expected outcomes at the time of transplantation, ${ }^{3-5}$ and implementation of the KAS has been associated with diminished 1-year patient and graft survival rates. ${ }^{6,7}$

The goal of our study was to identify how donor and recipient characteristics independent of each other would

Copyright @ 2019 by Thieme Medical Publishers, Inc., 333 Seventh Avenue, New York, NY 10001, USA. Tel: +1(212) 584-4662.
DOI https://doi.org/ 10.1055/s-0039-1700500. ISSN 1061-1711. 
influence graft survival. Our rationale was to be able to provide the recipient with an overall assessment of the expected kidney transplant survival at the time of the organ offer based on his/her characteristics and the characteristics of the donor (deceased or live), and to be able to determine what organ to choose in instances where several options were available.

\section{Methods}

Total 39,108 subjects who underwent kidney transplant between October 25, 1999 and January 1, 2007 were identified in the United Network for Organ Sharing (UNOS) dataset. Kidney recipients younger than 18 or with missing age and adult kidney recipients with multiple transplants were excluded. Our primary outcome was the graft survival. ${ }^{8}$ Two sets of covariates were of interest, one was donor characteristics and the other was recipient characteristics. ${ }^{1,9}$ According to the existing donor classifications, the Kidney Donor Risk Index (KDRI) and KDPI, donor age, height, weight, ethnicity (white, black, Hispanic, and other), history of hypertension, history of diabetes, cause of death (anoxia, cerebrovascular, head trauma, central nervous system tumor, others, and unknown), serum creatinine, hepatitis $C$ virus status, and donation after circulatory death were assessed (-Table 1). Regarding kidney transplant recipient characteristics, variables were selected based on both prior knowledge in literature, and included recipient age, gender, body mass index (BMI), history of previous kidney

Table 1 Donor demographic characteristics of the study population

\begin{tabular}{|c|c|c|c|}
\hline Characteristics & $\begin{array}{l}\text { No graft failure, } \\
n=30,063\end{array}$ & Graft failure, $n=9,045$ & $p$-Value \\
\hline \multicolumn{4}{|l|}{ Donor characteristics } \\
\hline Age, y, mean (SD) & $36.3(16.6)$ & $40.3(17.4)$ & $<0.001$ \\
\hline Height, cm, mean (SD) & $169.1(17.7)$ & $169.3(16.3)$ & 0.195 \\
\hline Weight, kg, mean (SD) & $76.0(23.0)$ & $76.6(22.2)$ & 0.036 \\
\hline \multicolumn{4}{|l|}{ Race, \% } \\
\hline White & $21,640(72.0)$ & $6,819(75.4)$ & \\
\hline Black & $3,428(11.4)$ & $1,010(11.2)$ & \\
\hline Hispanic & $4,069(13.5)$ & $976(10.8)$ & \\
\hline Other & $926(3.1)$ & $240(2.6)$ & $<0.001$ \\
\hline \multicolumn{4}{|l|}{ Hypertension, \% } \\
\hline No & $23,590(78.5)$ & $6,562(72.5)$ & \\
\hline Yes & $6,240(20.8)$ & $2,408(26.6)$ & \\
\hline Unknown & $233(0.8)$ & $75(0.8)$ & $<0.001$ \\
\hline \multicolumn{4}{|l|}{ DM, \% } \\
\hline No & $28,606(95.2)$ & $8,432(93.2)$ & \\
\hline Yes & $1,345(4.5)$ & $574(6.3)$ & \\
\hline Unknown & $112(0.4)$ & $39(0.4)$ & $<0.001$ \\
\hline \multicolumn{4}{|l|}{ Cause of death, \% } \\
\hline Anoxia & $3,831(12.7)$ & $1,023(11.3)$ & \\
\hline Cerebrovascular & $11,409(37.9)$ & $4,076(45.1)$ & \\
\hline Head trauma & $13,945(46.4)$ & $3,659(40.4)$ & \\
\hline Central nervous system tumor & $243(0.8)$ & $88(1.0)$ & \\
\hline Unknown & $635(2.1)$ & $199(2.2)$ & $<0.001$ \\
\hline Serum creatinine, mean (SD) & $1.03(0.5)$ & $1.03(0.5)$ & 0.412 \\
\hline Cold ischemia time, mean (SD) & $17.6(8.9)$ & $18.5(8.6)$ & $<0.001$ \\
\hline \multicolumn{4}{|l|}{$\mathrm{HCV}, \%$} \\
\hline No & $29,343(97.6)$ & $8,716(96.4)$ & \\
\hline Yes & $720(2.4)$ & $329(3.6)$ & $<0.001$ \\
\hline \multicolumn{4}{|l|}{ Donation after circulatory death, \% } \\
\hline No & $28,518(94.9)$ & $8,599(95.1)$ & \\
\hline Yes & $1,545(5.1)$ & 446 (4.9) & 0.429 \\
\hline
\end{tabular}

Abbreviations: DM, diabetes mellitus; HCV, hepatitis C virus; SD, standard deviation. 
transplant, previous malignancy, peripheral vascular disease, drug-treated chronic obstructive pulmonary disease, symptomatic cerebrovascular disease, hepatitis C status, dialysis at transplant, diabetes at registration, and education (-Table 2).

Demographic characteristics of the study population, stratifying by graft survival status, were present (- Tables $\mathbf{1}$ and $\mathbf{2}$ ). The Wilcoxon rank-sum test and the chi-squared test were used to compare differences between continuous variables, and categorical variable, respectively, for those who had graft failure versus who did not. The number of person-years of graft survival was calculated for each individual. The end point of the study included lost to follow-up, death, patient experienced graft failure, and end of follow-up. The Kaplan-Meier survival curve was presented for the entire cohort. Cox proportional hazard models were used to identify predictors of graft failure. Two multivariate Cox models were constructed for donor risk factors and recipient risk factors, respectively. Cox models with backward elimination were used for multivariate analysis of recipient characteristics related to the incidence of graft failure. Proportional hazard assumption was assessed based on the Schoenfeld residuals. Prognostic scores were then estimated from each model.

Time-dependent receiver operating characteristic (ROC) curves were computed from censored survival data using the Nearest Neighbor Estimation (NNE) method proposed by Heagerty et al. ${ }^{18}$ Estimated area under time-dependent ROC curve (AUC) was used to compare the predictive ability of the two allocation systems. The "survivalROC" package written using $\mathrm{R}$ was used to conduct the estimation of timedependent ROC curves. The rest of the analyses was performed in Stata 14.0.

Our study design was submitted to the institutional review board (IRB) at the Feinstein Institute for Medical Research at Northwell Health. The IRB determined that the study did not meet the definition of human subject research because it did not involve identifiable information, no data or specimens were collected, and there was no direct intervention or interaction. Thus, review or approval was not required by the IRB.

Table 2 Recipient demographic characteristics of the study population

\begin{tabular}{|l|l|l|l|}
\hline Recipient characteristics & No graft failure, $\boldsymbol{n}=\mathbf{3 0 , 0 6 3}$ & Graft failure, $\boldsymbol{n}=\mathbf{9 , 0 4 5}$ & $\boldsymbol{p}$-Value \\
\hline Age, $\mathrm{y}$, mean (SD) & $48.3(12.9)$ & $56.6(11.4)$ & $<0.001$ \\
\hline Gender, \% & & & \\
\hline Female & $12,258(40.8)$ & $3,274(36.2)$ & \\
\hline Male & $17,805(59.2)$ & $5,771(63.8)$ & $<0.001$ \\
\hline BMI, \% & & & \\
\hline Underweight & $840(2.8)$ & $202(2.2)$ & \\
\hline Normal & $11,285(37.5)$ & $3,043(33.6)$ & \\
\hline Overweight & $10,127(33.7)$ & $3,200(35.4)$ & $<0.001$ \\
\hline Obese & $7,811(26.0)$ & $2,600(28.7)$ & $<0.001$ \\
\hline History of previous kidney transplant, \% & $3,340(11.1)$ & $836(9.2)$ & $<0.001$ \\
\hline Previous malignancy, \% & $989(3.3)$ & $519(5.7)$ & $<0.001$ \\
\hline Peripheral vascular disease, \% & $916(3.1)$ & $694(7.7)$ & \\
\hline Drug-treated COPD, \% & & & \\
\hline No & $29,762(99.0)$ & $8,876(98.1)$ & \\
\hline Yes & $256(0.8)$ & $140(1.6)$ & $<0.001$ \\
\hline Unknown & $45(0.2)$ & $29(0.3)$ & 0.001 \\
\hline Current PRA & $9.9(23.7)$ & $9.0(22.5)$ & \\
\hline HCV, \% & & & $<0.001$ \\
\hline No & $25,154(83.7)$ & $7,388(81.7)$ & \\
\hline Yes & $1,722(5.7)$ & $720(8.0)$ & $<0.001$ \\
\hline Unknown & $3,187(10.6)$ & $937(10.4)$ & \\
\hline Dialysis at transplant, \% & $27,288(90.8)$ & $4,405(92.9)$ & \\
\hline DM, \% & $7,600(25.3)$ & $3,447(60.2)$ & \\
\hline Education, \% & & $17,147(57.0)$ & \\
\hline Below college & $12,916(43.0)$ & \\
\hline College or above & & \\
\hline
\end{tabular}

Abbreviations: BMI, body mass index; COPD, chronic obstructive pulmonary disease; DM, diabetes mellitus; HCV, hepatitis C virus; PRA, panel reactive antibody; SD, standard deviation. 


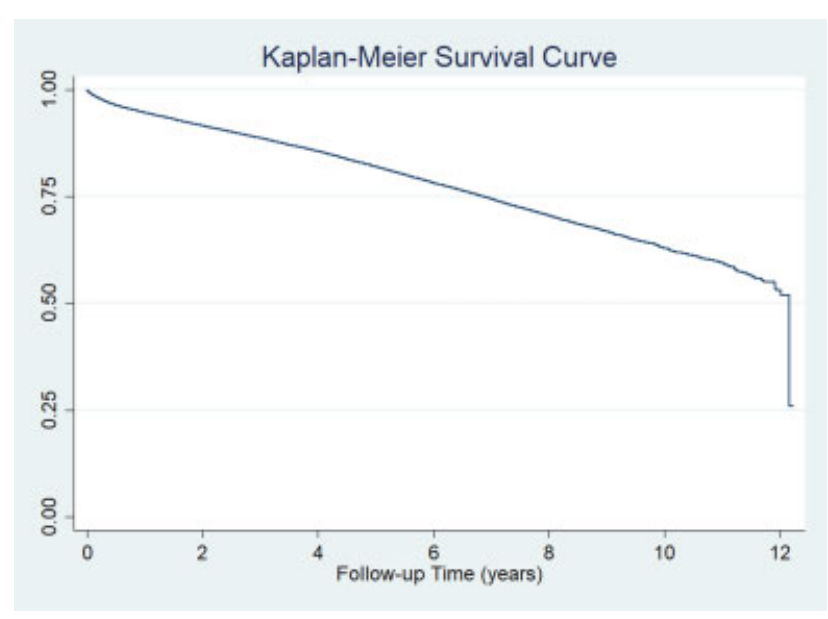

Fig. 1 Kaplan-Meier median survival curve.

\section{Results}

Among the 39108 subjects, 9,045 (23.1\%) experienced graft failure with a median follow-up time of 5.3 years. The median survival time was $\sim 12$ years ( - Fig. 1 ). The mean ( \pm standard deviation) individual prognostic scores from the donor characteristics model and recipient characteristic model were $19.8( \pm 11.1)$ and $18.9( \pm 14.7)$, respectively. According to the donor characteristics model, the 20-year survival rate was 0.153 and the estimated $\mathrm{AUC}(\mathrm{t})$ at 20 years was 0.673 , while the estimated $\mathrm{AUC}(\mathrm{t})$ at 20 years based on recipient model was 0.742 using NNE method (-Fig. 2).

In our donor model, age, height, serum creatinine, and donation after circulatory death did not achieve statistical significance. When considering recipient characteristics, all parameters evaluated achieved statistical significance. Further analysis ( - Table 3 ) showed that although BMI was statistically significant, the subcategory "overweight" did not reach significance on its own. The predictive value of the recipient model $(A U C=0.752)$ was greater than that of the donor model (AUC $=0.673$ ).

During the first year after transplantation, both donor and recipient models had identical relevance. From the first to

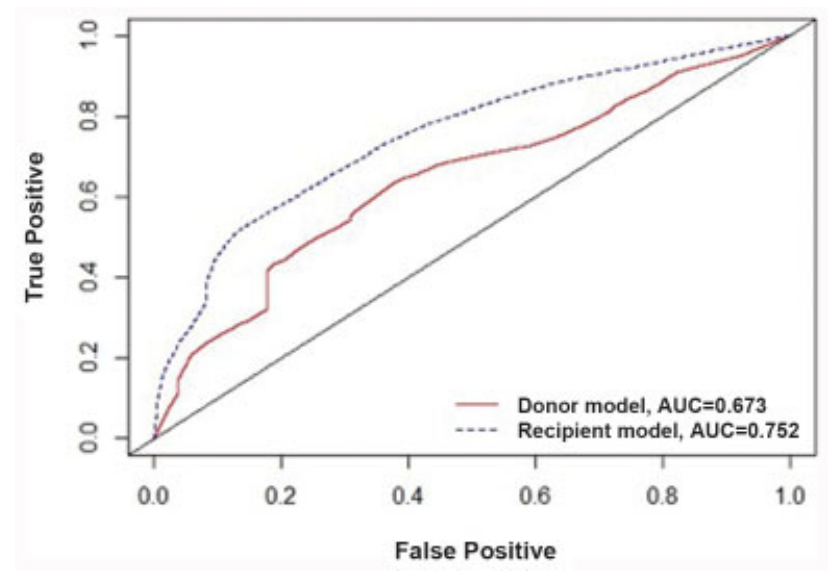

Fig. 2 Estimated receiver operating characteristic curve (t) at 20 years by two models, Nearest Neighbor Estimation method. AUC, area under the curve. the sixth years, although the two ROC curves were nearly identical, the donor model outweighed the recipient model. Both models intersected again at the sixth year. From that time onward, the difference between the two ROC curves increased and the ROC curve for recipient characteristics model predominated over the ROC curve for donor characteristics model (-Fig. 3).

\section{Discussion}

The KAS, based on the risk instruments KDPI/ KDRI (Kidney Donor Renal Index) and EPTS, was implemented on December 2014. 2,10 The KDRI (Kidney Donor Renal Index) is based on 10 donor characteristics (age, cause of death, creatinine level, diabetes, donation after cardiac death, ethnicity, height, hepatitis $\mathrm{C}$ serology, hypertension, weight). ${ }^{7,11}$ It provides the relative risk of failure after transplantation of a specific deceased donor graft with respect to the median kidney donor from the previous year. ${ }^{1}$ A KDRI of 1 represents the median (50th percentile) of previous calendar year donor. ${ }^{11}$ The KDPI provides a percentage risk scale for each individual deceased donor based on the relative risk of the KDRI. ${ }^{1}$ It eliminates transplant-related factors from the KDPRI and is normalized to a percentile value. ${ }^{12} \mathrm{~A}$ donor with a KDPI of $20 \%$, for example, has a KDRI that exceeds at least $19 \%$ and at most $20 \%$ of the entire donor reference population. $^{2}$

The EPTS is a score ranging from 0 to $100 \%$ that is assigned to all adult candidates on the kidney transplant waiting list based on time on dialysis, diagnosis of diabetes, prior solid organ transplant, and age. ${ }^{13}$ Recipients with EPTS $\leq 20 \%$ receive priority for kidneys from donors with KDPI $\leq 20 \%{ }^{2,9-11}$

The incidence of graft failure in our study was $23.1 \%$ at 5.3 years. Both donor and recipient models had identical relevance during the first posttransplant year. From the first to the sixth year, although the two ROC curves were nearly identical the donor model outweighed the recipient model. After the sixth year, the difference between the two ROC curves increased and the ROC curve for recipient characteristics model predominated over the ROC curve for donor characteristics model. These findings could facilitate the allocation of organs based on both donor and recipient characteristics and the improved utilization of marginal organs.

The current 5-year graft survival is $57.6 \%$ for organs with KDPI $>85 \%$, and $73.3 \%$ for those with KDPI 35 to $85 \% .{ }^{14}$ Transplantation of high KDPI deceased donor kidneys was found to be associated with a greater short-term but a lower long-term recipient mortality risk when compared with nontransplantation based on the expectation of receiving an organ with a lower KDPI. ${ }^{12}$ The magnitude of the benefit decreased after the 4 th posttransplant year. ${ }^{12}$ The cumulative survival "break-even point" for transplantation with high KDPI kidneys was 7.7 months for KDPI scores of 71 to $80,18.0$ months for scores 81 to 90 , and 19.8 months for 91 to $100 .^{12}$ The greatest survival benefit was observed among recipients either $>50$ years of age or with a median wait time 
Table 3 Association of recipient characteristics and graft failure

\begin{tabular}{|c|c|c|c|}
\hline Recipient & HR & $95 \% \mathrm{Cl}$ & $p$-Value \\
\hline Age & 1.042 & $1.040,1.043$ & $<0.001$ \\
\hline \multicolumn{4}{|l|}{ Gender } \\
\hline Female & Ref. & & \\
\hline Male & 1.109 & $1.077,1.142$ & $<0.001$ \\
\hline \multicolumn{4}{|l|}{ BMI } \\
\hline Normal & Ref. & & \\
\hline Underweight & 1.296 & $1.190,1.412$ & $<0.001$ \\
\hline Overweight & 0.981 & $0.950,1.014$ & 0.260 \\
\hline Obese & 1.084 & $1.047,1.123$ & $<0.001$ \\
\hline History of previous kidney transplant & 1.221 & $1.162,1.283$ & $<0.001$ \\
\hline Previous malignancy & 1.171 & $1.108,1.239$ & $<0.001$ \\
\hline Peripheral vascular disease & 1.360 & $1.288,1.437$ & $<0.001$ \\
\hline Drug-treated COPD & 1.306 & $1.197,1.425$ & $<0.001$ \\
\hline Current PRA & 1.001 & $1.000,1.002$ & 0.002 \\
\hline \multicolumn{4}{|l|}{$\mathrm{HCV}, \%$} \\
\hline No & Ref. & & \\
\hline Yes & 1.532 & $1.455,1.613$ & $<0.001$ \\
\hline Unknown & 1.084 & $1.029,1.142$ & 0.002 \\
\hline Dialysis at transplant & 1.506 & $1.430,1.585$ & $<0.001$ \\
\hline DM & 1.619 & $1.574,1.665$ & $<0.001$ \\
\hline \multicolumn{4}{|l|}{ Education } \\
\hline Below college & Ref. & & \\
\hline College or above & 0.863 & $0.840,0.887$ & $<0.001$ \\
\hline Cold ischemia time & 1.005 & $1.004,1.007$ & $<0.001$ \\
\hline
\end{tabular}

Abbreviations: $\mathrm{BMI}$, body mass index; $\mathrm{Cl}$, confidence interval; COPD, chronic obstructive pulmonary disease; DM, diabetes mellitus; HR, hazard ratio; $\mathrm{HCV}$, hepatitis C virus; PRA, panel reactive antibody.

$\geq 33$ months for KDPI 71 to $80 \%$ or with both characteristics for KDPI 81 to $100 \% .^{12}$ Both short- and long-term timeassociated interactions become of greater relevance as the proportion of candidates 65 years of age or older continues to increase and will likely surpass those aged between 35 and 49 by $2020 .^{5,14}$ Our results are consistent with the findings

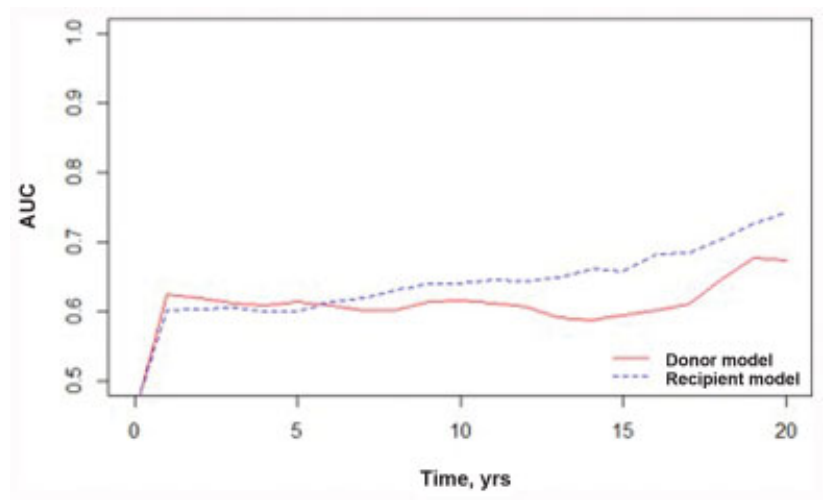

Fig. 3 Area under the curve $(A \cup C(t))$ based on donor and recipient model for the first 20 years. by Calvillo-Arbizu J et al, which focused on the odds of death and graft loss based on organ quality as measured by KDPI. The authors showed that the hazard survival ratio for patients over 60 years old was lower than that among those aged between 18 and 59 years. ${ }^{15}$

The current kidney discard rate is $29.6 \%$ for donors who died of cerebrovascular accidents, $32.9 \%$ for donors aged between 50 and $64,36.3 \%$ for donors with terminal creatinine $>1.5 \mathrm{mg} / \mathrm{dL}, 36.6 \%$ for donors with hypertension, $45.1 \%$ for donors with diabetes, $59.1 \%$ for donors with KDPI $>85 \%$, and $62.3 \%$ for donors $\geq 65$. ${ }^{14}$ Our model expands the number of variables of the EPTS, which can be applied to the time of the organ offer to avoid continuous deterioration of potential recipients. Data show an increase in the number of candidates removed from the waitlist because of worsening medical conditions. ${ }^{14,16}$

Although more predictive of donor quality than the previous binary expanded versus standard criteria donor classification, KDRI has only a "moderately predictive" c-statistic power of $\sim 0.60 .^{2,6,7}$ It does not take into consideration recipient or donor/recipient characteristics and its predictive power is greatest at both extremes. ${ }^{11}$ Furthermore, it has been 
associated with worse overall patient survival at one year as well as a trend towards increased graft outcomes. ${ }^{17}$

In our study, we propose a predictive model that evaluates the dynamic effect and relevance of both donor and recipient characteristics after transplantation. As opposed to the KDPI tables that use logistic regression analysis assuming a fixed time, our model incorporates the concept of evolving risk over time. We also further define the short, intermediate, and longterm interaction of both donor and recipient characteristics on kidney graft survival. We do not assume a constant risk, and base our calculations on a Cox model that takes time into account. Although we used the same variables than KDPI, our study had a better predictive value than that of the KDPI. Our predictive value for recipient characteristics was also better than the predictive value of the KDPI for donor characteristics.

The survivalROC package is a useful tool in computing time-dependent $\mathrm{ROC}(\mathrm{t})$ curves, but has several limitations. It does not take time-varying covariates into account. It does not calculate confidence intervals of AUCs either, and therefore we cannot compare whether there is a statistically significant difference between the two markers measured on the same subjects. Like all other studies based on databases, we are also limited to the variables gathered by UNOS, such as a reporting bias associated with rapid notification of critical events (death or graft failure) and delayed notification of continued survival.

We hope that this model will provide additional guidance and risk stratification by further optimizing organ allocation and decreasing organ discard in deceased donor kidney transplantation.

\section{Author Contribution}

Study conception and design: JY, CLSM, and EPM. Acquisition of data: JY, CLSM, HD, RR, and EPM. Analysis and interpretation of data: JY, CLSM, JC, EG, HR, LWT, EPM. Drafting of manuscript: JY, CLSM, JC, HD, and EPM. Critical revision of the manuscript: CLSM, JC, EG, HR, LWT, EPM.

\section{Conflict of Interest Statement}

The authors declare that the research was conducted in the absence of any commercial or financial relationship that could be construed as a potential conflict of interest.

\section{Acknowledgments}

This work was supported in part by the Health Resources and Services Administration contract 231-00-0015. The content is the responsibility of the authors alone and does not necessarily reflect the views or policies of the Department of Health and Human Services, nor does mention of trade names, commercial products, or organizations imply endorsement by the US government.

\section{References}

1 Organ Procurement and Transplantation Network (OPTN): KDRI to KDPI Mapping Table. Available from: https://optn.transplant.hrsa. gov/media/2150/kdpimapping_table.pdf [accessed 02.10.19]

2 Organ Procurement and Transplant Network. A Guide to Calculating and Interpreting the Kidney Donor Profile Index (KDPI). https://optn.transplant.hrsa.gov/media/1512/guide_to_calculating_interpreting_kdpi.pdf (13 February 2019, date last accessed)

3 Heilman RL, Green EP, Reddy KS, Moss A, Kaplan B. Potential impact of risk and loss aversion on the process of accepting kidneys for transplantation. Transplantation 2017;101(07): 1514-1517

4 Tversky A, Kahneman D. Advances in prospect theory: cumulative representation of uncertainty. J Risk Uncertain 1992;5:297-323

5 Hellemans R, Stel VS, Jager KJ, Bosmans JL, Abramowicz D. Do elderly recipients really benefit from kidney transplantation? Transplant Rev (Orlando) 2015;29(04):197-201

6 Clayton PA, McDonald SP, Snyder JJ, Salkowski N, Chadban SJ. External validation of the estimated posttransplant survival score for allocation of deceased donor kidneys in the United States. Am J Transplant 2014;14(08):1922-1926

7 Rao PS, Schaubel DE, Guidinger MK, et al. A comprehensive risk quantification score for deceased donor kidneys: the Kidney Donor Risk Index. Transplantation 2009;88(02):231-236

8 Hariharan S, Johnson CP, Bresnahan BA, Taranto SE, McIntosh MJ, Stablein D. Improved graft survival after renal transplantation in the United States, 1988 to 1996. N Engl J Med 2000;342(09): 605-612

9 KDPI Calculator: Organ Procurement and Transplantation Network; https://optn.transplant.hrsa.gov/media/1512/guide_to_calculating_ interpreting_kdpi.pdf. Accessed January 2019

10 Massie $A B$, Luo $X$, Lonze $B E$, et al. Early changes in kidney distribution under the new allocation system. J Am Soc Nephrol 2016;27(08):2495-2501

11 Lee AP, Abramowicz D. Is the Kidney Donor Risk Index a step forward in the assessment of deceased donor kidney quality? Nephrol Dial Transplant 2015;30(08):1285-1290

12 Massie AB, Luo X, Chow EK, Alejo JL, Desai NM, Segev DL. Survival benefit of primary deceased donor transplantation with highKDPI kidneys. Am J Transplant 2014;14(10):2310-2316

13 Merion RM, Ashby VB, Wolfe RA, et al. Deceased-donor characteristics and the survival benefit of kidney transplantation. JAMA 2005;294(21):2726-2733

14 Hart A, Smith JM, Skeans MA, et al. OPTN/SRTR 2015 annual data report: kidney. Am J Transplant 2017;17(Suppl 1):21-116

15 Calvillo-Arbizu J, Pérez-Valdivia MA, Gentil-Govantes MA, et al. Does the Kidney Donor Profile Index (KDPI) predict graft and patient survival in a Spanish population? Nefrologia 2018;38(06): 587-595

16 Wachterman MW, Marcantonio ER, Davis RB, et al. Relationship between the prognostic expectations of seriously ill patients undergoing hemodialysis and their nephrologists. JAMA Intern Med 2013;173(13):1206-1214

17 Wilk A, Beck J, Kucheryavaya A. The Kidney Allocation System (KAS): The First Two Years. Richmond, Virginia: Organ Procurement and Transplantation Network (OPTN) Kidney Transplantation Committee; 2017

18 Heagerty PJ, Lumley T, Pepe MS. Time-dependent ROC curves for censored survival data and a diagnostic marker. Biometrics 2000; 56(02):337-44 